\title{
The Anisotropy in the distribution of Extragalactic Infrared Sources and Background.
}

\author{
P.de Bernardis, R. Fabbri ${ }^{0}$, S.Masi, F.Melchiorri,B. Olivo ${ }^{+}$, W.Pecorella \\ Dipartimento di Fisica, Università "La Sapienza", Roma , Italy \\ ${ }^{0}$ Dipartimento di Fisica, Università degli studi , Firenze , Italy \\ + IFA-CNR, Roma, Italy
}

\section{Abstract}

We analyze the anisotropies in the extragalactic infrared background and in the source counts in standard cosmology perturbed by large-scale, small-amplitude density fluctuations. The dipole anisotropy of the diffuse background is connected to the dipole of the cosmic background radiation and of the source counts, and a full consistency with a large $(\simeq 7 \%)$ anisotropy in the IRAS source counts is found to imply a low density $\left(\Omega_{0} \simeq 0.2\right)$ universe, contrary to a previous claim. We analyze also higher order harmonics. Using the IRAS Low-Resolution All-Sky Maps we obtain an upper limit of $0.17 \mathrm{MJy} / \mathrm{sr}$ on the dipole of the $100 \mu \mathrm{m}$ background and show that it is consistent with a source-count anisotropy of $7 \%$ only for a low intensity, $I \leq 1.2 \mathrm{MJy} / \mathrm{sr}$, of the background itself.

\section{INTRODUCTION}

Distant, unresolved IR galaxies and quasars are expected to generate an infrared diffuse background (Tinsley (1973), Bond et al.(1986) and Fabbri et al.(1987)). From the analysis of the IRAS Low-Resolution All-Sky Maps (see IRAS Explanatory Supplement(1984)), Rowan-Robinson (1986) inferred the existence of a $100 \mu m$ isotropic background of intensity $I=5.7 \pm 2.5 \mathrm{MJy} / \mathrm{sr}$. At the same time, from the IRAS Point Source Catalog Meiksin and Davis (1986) and Yahil et al.(1986) derived a dipole anisotropy of $2-7 \%$ in the extragalactic source counts at 60 and $100 \mu \mathrm{m}$, the maximum direction being in reasonable agreement with the dipole of the cosmic background radiation (CBR). From a comparison of the CBR and IR-source-counts dipoles and using a simple model where the cosmic expansion is neglected, Yahil et al.(1986) provided an independent estimate of the cosmological density parameter $\Omega_{0}$. Here we provide a more satisfactory, 15

J. Audouze et al. (eds.), Large Scale Structures of the Universe, 15-23.

(c) 1988 by the IAU. 
comparative analysis of the large-scale anisotropies of the IR extragalactic background, the IR source counts and the CBR. Performing the harmonic expansion of the anisotropies in Friedmann models perturbed by long-wave, small amplitude density fluctuations, we derive a number of fairly general results. The only relevant assumption is that the dominant contribution to the anisotropies comes from perturbation scales well inside the particle horizon, which is true for all the perturbation spectra considered in current theories of galaxy formation. First we show that the IR background dipole amplitude $(\Delta I / I)_{1}$ is connected to the CBR dipole $(\Delta T / T)_{1}$ by

$$
(\Delta I / I)_{1}=F\left(\alpha, \Omega_{0}\right)(\Delta T / T)_{1}
$$

with $\alpha$ the spectral index of the unresolved sources. A previous calculation of Yahil et al.(1986), neglecting the cosmic expansion effects, could not evidentiate the $\alpha$ - dependence of $\mathrm{F}$ and lead to a questionable value of $\Omega_{0}$. Here we show that, for any reasonable value of $\alpha$, the comparison of the CBR and the IR dipoles leads to $\Omega_{0} \simeq 0.2$ if we attribute the bulk of the IR count anisotropy to genuine large-scale effects.

Further, we show that for the measured luminosity function of IR sources (Lawrence et al.(1986)) we have for each harmonic amplitude

$$
(\Delta N / N)_{l} \simeq(\Delta I / I)_{l}
$$

where $(\Delta N / N)_{l}$ is the source-counts anisotropy of 1 -th order. If the available data on $(\Delta T / T)_{1}$ and $(\Delta N / N)_{1}$ contain genuine cosmological effects, then eqs.(1) and (2) provide two independent estimates of the IR background dipole anisotropy, which should be of order several percents. Also, independently of the specific perturbation spectrum, the anisotropies as functions of the angular scale $\gamma$ are given by $\Delta I / I \simeq$ $\Delta N / N \propto \gamma^{-1 / 2}$. Therefore we expect to find a quite chaotic IR background $(\Delta I / I>10 \%)$ at scales of order a few degrees. The large intensity of the IR dipole itself has an important consequence. From either (1) or (2), the absolute value of the IR background can be extimated if the dipole amplitude $\Delta I_{1}$ is known. Since the latter quantity has not yet been measured, in sect.III we use the IRAS Low-Resolution All-Sky Maps searching for a background dipole. Our result at $100 \mu \mathrm{mis}$

$$
\Delta I_{1} \leq 0.17 \mathrm{MJy} / \mathrm{sr}
$$

(3) From eq.(1) with $\alpha=-2$ (this choice is consistent with Ennis and Neugebauer (1985), Low and Tucker (1968)) and $\Omega_{0} \leq 1$, we get $I \leq 2.7 M J y / s r$. From eq.(2) with $\sqrt{3 / 4 \pi} \times(\Delta N / N)_{1}=0.07$ we get 
$I \leq 1.2 \mathrm{MJ} y / \mathrm{sr}$. Note that the latter limit is also derived from eq.(1) if one assumes the above mentioned value for the cosmic density, $\Omega \simeq 0.2$. Both limits contraddict the value claimed by Rowan-Robinson (1986) for the $100 \mathrm{~m}$ extragalactic background.

\section{THEORETICAL FRAMEWORK}

In this section we outline a unified description of the large-scale anisotropies in the integrated background and source counts, assuming that a Friedmann cosmology is perturbed by small-amplitude density fluctuations on scales greater than several tens of Megaparsec. The unperturbed spacetime metric is written as

$$
d s^{2}=R^{2}(\eta)\left[-d \eta^{2}+d \chi^{2}+\Sigma^{2}(\chi)\left(d \Theta^{2}+\sin ^{2} \Theta d \phi^{2}\right)\right],
$$

where $\Sigma(\chi)=K^{-1 / 2} \sin \left(K^{1 / 2} \chi\right)$, and $\mathrm{K}=+1,0,-1$ denotes the sign of spatial curvature. The density fluctuation $\Delta \rho / \rho$, treated in the linear regime, is suitably expanded in a series of eigenfunctions of wavenumber $k=2 \pi R / \lambda$ and of angular momentum,

$$
\Delta \rho / \rho=\sum_{l, m} \int d k\left(k^{2}-4 k\right) C_{k l m} \delta_{k l}(\chi, \eta) Y_{m l}(\Theta, \phi)
$$

(cfr. Lifshitz and Khalatnikov (1963)). We further assume that the large-scale distribution of sources traces the overall matter distribution, so that the source number density will be proportional to $(1+z)^{3} \chi(z)$ $(1+\Delta \rho / \rho)$ (with $\chi(z)$ taking into account evolutive effects). Observable anisotropies are generated by the source inhomogeneities, and also by the metric perturbation associated to the cosmic density fluctuations. Fabbri et al.(1987a) provide formulas for the anisotropy of the integrated background on the assumption that all the sources exhibit the same spectral function $\exp \int^{\nu} \alpha d \nu$, with $\alpha$ the spectral index. Expanding the anisotropic part of I in spherical harmonics,

$$
\Delta I / I=(4 \pi)^{-1 / 2} \sum_{l, m}(\Delta I / I)_{l m} Y_{l m}(\Theta, \phi)
$$

we have

$$
\begin{gathered}
(\Delta I / I)_{l m}=\int d k C_{k l m} I_{k} \\
I_{k l}=\int\left[\delta_{k l}\left(\chi, \eta_{0}-\chi\right)+(3-\alpha) \Delta_{k l}(\chi)\right] F_{l}(\nu, \chi) d \chi
\end{gathered}
$$


where $\eta_{0}$ denotes the present epoch, $\mathrm{F}$ is a weighting function

$$
F(\nu, \chi)=\frac{\left\{\left[\exp \int^{\nu(1+z)} \alpha\left(\nu^{\prime}\right) d \nu^{\prime}\right] \chi(z) /(1+z)\right\}}{\left\{\int^{\nu(1+z)}\left[\exp \int \alpha\left(\nu^{\prime}\right) d \nu^{\prime}\right] \chi(z) /(1+z) d \chi\right\}}
$$

and $\Delta_{k l}(\chi)$ takes into account Doppler and gravitational shifts and is calculated in Fabbri et al.(1987a). For the CBR, we simply have $I_{k l}=\Delta_{k l}\left(\chi_{E}\right)$, where $\chi_{E}$ is the radial coordinate evaluated at the last scattering, $z=10^{3}$.

Analogous equations can be found for the angular dependence of $N(S)$, the number of sources (per unit solid angle) with observed monochromatic flux S at frequency $\nu$. Expanding its anisotropic part $\Delta N / N$ in spherical harmonics, one can define suitable coefficients $N_{k l}$

quite analogous to the $I_{k l}$ of eq.(7), which turn out to be given by

$$
N_{k l}=\int\left[\delta_{k l}\left(\chi, \eta_{0}-\chi\right)-(1+s)(3-\alpha) \Delta_{k l}\right] F_{N}(\chi, S, \nu) d \chi
$$

where $F_{N}$ can be expressed in terms of the luminosity function $\Phi(L)$,

$$
F_{N}(\chi, S, \nu)=\frac{\left\{L \Phi(L) \chi(z) \Sigma^{2}(\chi)\right\}}{\int L \Phi(L) \chi(z) \Sigma^{2}(\chi) d \chi}
$$

and $s=d \ln \Phi / d \ln L$. (Note that $L(\chi, S)$ is the intrinsic luminosity of a source which, placed at a coordinate distance $\chi$, is observed to show a monochromatic flux S). For the derivation of eqs.(9) and (10) see Fabbri et al.(1987b). Since both $I_{k l}$ and $N_{k l}$ do not depend on the azimuthal eigenvalue m, for each value of 1 we get the same angular pattern in $\Delta I_{k l} / I$ and $\Delta N / N$. Thus for instance, both IR dipoles should be parallel to the CBR dipole. Further one can show that for $\mathrm{s}=2$ we have $F_{N}=F_{l}$ and therefore $N_{k l}=I_{k l}$ for all values of $\mathrm{k}$ and $l$. As an example, in fig. 1 we report the computed values of such coefficients for 1 $=1,2$ for sources with $\chi(z)=1$ and $\alpha=-1,-2,-3$, in a background cosmology with $\Omega_{0}=0.1$. It is easy to check that for $k \gg 2 \pi$ (perturbation scales well inside the particle horizon) all the coefficients are simply proportional to $\mathrm{k}$. This result holds for any values of $\alpha$ and $\Omega_{0}$; for $\Omega_{0}=1$ we have the simple analytic formula:

$$
N_{k l}=I_{k l}=(k / 10)\left[(3-2 \alpha)(l-1) ! !(\pi / 2)^{\left[1+(-1)^{l}\right] / 2} / l ! !+2(3-\alpha) \delta_{l 1} / 3\right]
$$

For $s \geq-2$, one can show (Fabbri et al.(1987b)) that $N_{k l} \leq I_{k l}$. The best value for $s$ in the lowluminosity region which dominates the integrals, is $s=-1.8_{-0.10}^{+0.05}$ (Lawrence et al.(1986)). 


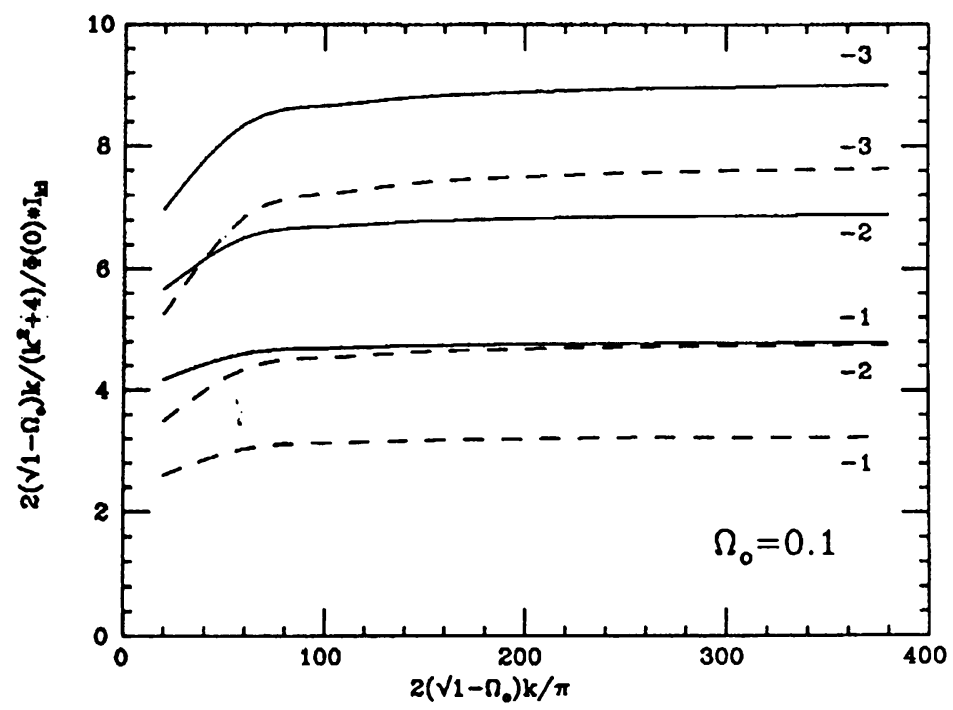

Figure 1: The coefficients $I_{k 1}$ (full lines) and $I_{k 2}$ (dashed) for an IR background originated by sources with $\chi=1$. The cosmic density parameter is taken as $\Omega_{0}=0.1$. The curves are labelled by the corresponding values of the spectral index $\alpha$, assumed to be constant.

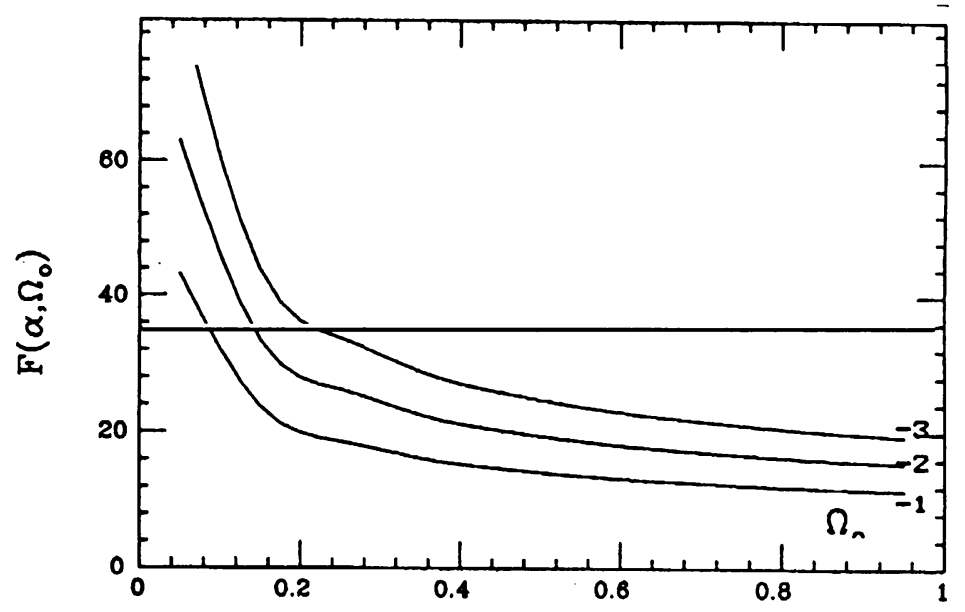

Figure 2: The ratio of the IR dipole to the CBR dipole as a function of $\Omega_{0}$, for selected values of $\alpha$. The horizontal line $\mathrm{F}=35$ corresponding to $\sqrt{3 / 4 \pi}(\Delta N / N)_{1}=0.07$ crosses the theoretical curves for $\Omega_{0} \simeq 0.2$. 
Thus we find that the equation $N_{k l}=I_{k l}$ should hold in practice with a good accuracy. This result for $\mathrm{l}=1$ provides an estimate of $(\Delta I / I)_{1}$ on the grounds that $\sqrt{(3 / 4 \pi)}(\Delta N / N)_{1} \simeq 2-7 \%$. In current theories of galaxy formation, the perturbations are described by spectra $\delta(k)^{2} \propto k^{n}, n \geq-1$ (Silk and Wilson (1985)). The expected anisotropies are then

$$
(\Delta I / I)_{l}^{2}=\sum_{m}(\Delta I / I)_{l m}^{2}=\operatorname{const}(2 l+1) \int d k k \delta(k)^{2} I_{k l}^{2} /\left(k^{2}-4 K\right)^{2}
$$

An analogous equation is obtained for $(\Delta N / N)_{l}$ replacing $I_{k l}-->N_{k l}$. The integral in eq.(12) is dominated by the large-k region. Thus for the dipole we have

$$
F\left(\alpha, \Omega_{0}\right)=\lim _{k \rightarrow \infty}\left(I_{k 1} / I_{k 1}^{C B R}\right)
$$

with $F\left(\alpha, \Omega_{0}\right)$ defined by eq.(1). In fig.(2) we report $F\left(\alpha, \Omega_{0}\right)$ as a function of $\Omega_{0}$ for some values $\alpha$. The limit value for $\Omega \rightarrow 1$ is $F(\alpha, 1)=(15-8 \alpha) / 2$. Since the expected value of $\alpha(\lambda /(1+z))$ at $\lambda \simeq 100 \mu \mathrm{m}$ is -2 (Ennis and Neugebauer,(1985),Low(1968)), then $F>15$. The measured value of the CBR dipole is $(\sqrt{3 / 4 \pi})(\Delta T / T)_{1} \simeq 1.2 \times 10^{-3}$ (Lubin et al.(1985),Fabbri et al.(1980)) but the correction for the local velocity field effects leads to $\simeq 2.0 \times 10^{-3}$ (Rubin et al.(1976), Peterson et al.(1986), Collins et al.(1986), Burnstein et al.(1986), Aaronson et al.(1986)). Thus we estimate $(\Delta I / I)_{1} \geq 3 \%$. Further, if we identify $(\Delta I / I)_{1}$ with $(\Delta N / N)_{1}$ we derive $\mathrm{F}=35$. Then fig.(2) shows that $\Omega_{0} \leq 0.2$.

Applying eq.(12) to higher-order harmonics in $\Omega_{0}=1$ models, we get for $l \geq 2$ and for any $n \geq-1$ :

$$
(\Delta I / I)_{l}^{2} /(\Delta I / I)_{1}^{2}=[(3-2 \alpha) /(5-8 \alpha / 3)]^{2}
$$

Using standard equations (e.g. Fabbri,Lucchin,Matarrese(1987)), one can calculate the rms anisotropy at the anoular scale $\gamma$. This is given by $\Delta I / I(\gamma) \propto \gamma^{-1 / 2}$.

\section{ANALYSIS OF IRAS AND BALLOON DATA}

In order to evaluate $\Delta I_{1}$, we used the IRAS Survey Low-Resolution All-Sky maps. Although $\Delta I_{1}$ is much smaller than the absolute intensity I of the extragalactic background, it has a characteristic spatial signature and can be recovered from the local background using a best fit procedure. This method is, in principle, independent of the zero-point calibration, which is always a difficult task for DC coupled systems. In fact the offset uncertainty is estimated to be $2.5 \mathrm{MJy} / \mathrm{sr}$ for the IRAS $100 \mu \mathrm{m}$ channel. Since we are interested in very large scale structures, we averaged the original $1 / 2^{0} \times 1 / 2^{0}$ IRAS data of the first sky 
coverage into $16545^{0} \times 5^{0}$ square pixels: the pixel array consisted of 36 pixel rows with $5^{0}$ galactic latitude width; the number of pixels for each row depends on the row's latitude in such a way that the solid angle of all the pixels is the same within 5\%. (In order to avoid projection distortions we used the maps centered at $l=0^{\circ}, b=0^{\circ}$ for the pixels between $-90^{\circ}$ and $90^{\circ}$ in galactic longitude, and the maps centered at $l=180^{\circ}, b=0^{\circ}$ for pixels outside this range). To handle with local emission (which cannot be completely well modelled by simple laws), we selected only two regions where the intensity is within 10the minimum in each galactic hemisphere. These regions are located symmetrically in the two hemispheres and are centered roughly half the way between ecliptic and galactic poles. They contain about 70 independent $5^{0} \times 5^{0}$ pixels. We performed a dipole fit of the IRAS data using the relation

$$
S=I_{0}+(\sqrt{3 / 4 \pi}) \Delta I_{1} \cos \Theta
$$

where $I_{0}$ is an offset constant and $\Theta$ is the angle between the CBR dipole direction and the IRAS line of sight; we found the following results: at $\lambda=60 \mu m, I_{0}=(7.60 \pm 0.02) \mathrm{MJy} / \mathrm{sr}, \Delta I=(0.47 \pm$ $0.17) \mathrm{MJy} / \mathrm{sr}, \chi^{2} / D O F=2.24 ;$ at $\lambda=100 \mu m, I_{0}=(6.31 \pm 0.03) \mathrm{MJy} / \mathrm{sr}, \Delta I=(0.018 \pm 0.067) \mathrm{MJy} / \mathrm{sr}$, $\chi^{2} / D O F=0.86$. We also investigated the possible subtraction of galactic emission in such regions, but neither the logarithm of galaxy counts nor the simple $\operatorname{cosec}(b)$ and $\operatorname{cosec}(\beta)$ laws describe atequately the data. We are then forced to consider the dipole best-fit as $1 \sigma$ upper limits to the dipole anisotropy of extragalactic backgrounds: from IRAS low resolution data we have $\Delta I(60 \mu m) \leq 0.64 \mathrm{MJy} / \mathrm{sr} ; \Delta I(100 \mu m) \leq$ $0.085 \mathrm{MJy} / \mathrm{sr}$. A balloon borne experiment devoted to the search for galactic and extragalactic radiation in the far infrared has been described by de Bernardis et al. (1984); in the low frequency channel ( $\lambda \geq 400 \mu m$ ) a dipole anisotropy was found after removing the galactic emission using a $\operatorname{cosec}(\mathrm{b})$ law. The direction of such a dipole is consistent with that of CBR dipole anisotropy but the amplitude is significantly larger. This would suggest the presence of an extragalactic background in the $800 \mu \mathrm{m}$ region with a dipole anisotropy of about $5 \times 10^{-13} \mathrm{Wcm}^{-2} \mathrm{sr}^{-1}$. A carefull reanalysis and recalibration of the data is in progress.

\section{CONCLUSIONS}

We have shown that two theoretical estimates are possible for the dipole anisotropy of the IR extragalactic background, based on the CBR dipole (eq.(1)) and on the IR-source counts (eq.(2)). Although both lead us to conclude that $(\Delta I / I)_{1} \geq 3 \%$, a complete agreement is obtained only for $\Omega_{0} \leq 0.2$, if $(\Delta N / N)_{1} \geq 7 \%$. This disagrees with Yahil et al.(1986). Within the limits of the linear theory, larger 
values of $\Omega_{0}$ would still be compatible with a large $(\Delta N / N)_{1}$ only for quite unrealistic values of the source spectral index; thus, substantial corrections to our results may come only in the case that $(\Delta N / N)_{1}$ has a nearby origin, where the linear treatment is inadeguate ( a mild nonlinearity would only cause small corrections, cfr. Yahil et al.(1986)). The analysis of dipole anisotropy of the IR background from the IRAS data, performed in sect.3, is consistent with the dipole of the source number counts only if the isotropic $\mathbb{R}$ background is sufficiently weak, $\mathrm{I}<1.2 \mathrm{MJy} / \mathrm{sr}$, which disagrees with Rowan-Robinson (1986). Therefore we are left with the following possibilities (i) the IR background claimed at a level of $5.7 \mathrm{MJy} / \mathrm{sr}$ is still of local origin, as supported by de Bernardis et al.(1987); or (ii) the bulk of the measured anisotropy $(\Delta N / N)_{1}$ is originated from scales $<10 \mathrm{Mpc}$. This is consistent with the fact that the IR count dipole appears to decrease for decreasing source flux (Meiksin and Davis(1986)).

\section{Aknowledgments}

This work has been supported by Piano Spaziale Nazionale of CNR

\section{References}

-Aaronson M., Bothun G., Mould J., Huchra J., Schomner R.A., Cornell M.E., 1986 , Ap.J. ,302,536

-Bond J.R., Carr B.J., Hogan C.J. 1986 ,Ap.J. ,306,428

-Burnstein D., Davies R.L., Dressler A., Faber S., Lynden-Bell D., Terlevich R., Wegner G., 1986 , Proc.Hawaii Conference on the Peculiar Velocity Field of the Universe, in press

-Collins A., Joseph R.D., Robertson N.A. 1986,Nature , 320,506

-de Bernardis P., Masi S., Melchiorri B., Melchiorri F., Moreno G.,1984, Ap.J.,278,150

-de Bernardis P., Masi S., Melchiorri F., Moreno G., Vannoni R., Aiello S. 1987 , submitted to Ap.J.

-Ennis D.J., Neugebauer G., 1982 , Ap.J. ,267, 460

-Fabbri R., Lucchin F., Matarrese S. ,1987 , Ap.J. ,315, 1

-Fabbri R., Andreani P., Nisini B., Melchiorri F., 1987a ,Ap.J. ,315,12

-Fabbri R., de Bernardis P., Masi S., Melchiorri F. ,1987b , in preparation

-Fabbri R., Guidi I., Melchiorri F., Natale V. ,1980 ,Phys.Rev.Lett. ,44,1563

-IRAS Explanatory Supplement 1984 , (Beichman C.A., Neugebauer G., Habing H.J., Clegg P.E., Chester T.J., eds.) JPL Publ. D-1855

-Lawrence A., Walker D., Rowan-Robinson M., Leech K.J., Penston M.V. 1986,MNRAS , ,687

-Lifshitz E.M., Khalatnikov I.M. 1963 ,Adv.Phys. ,12,185 
-Low F.J., Tucker W.H., 1968 , Phys.Rev.Lett. ,21, 1538

-Lubin P., Villela T., Epstein G., Smoot G. 1985 ,Ap.J.(Letters) ,298,L1

-Meiksin A., Davis M. 1986 ,Astron.J. ,91,191

-Peterson C.J. and Baumgart C.W. , Astron.J. ,91,530

-Rowan-Robinson M. 1986 ,MNRAS ,219,737

-Rubin V.C., Ford W.K.Jr., Thonnard N., Roberts M.S., Graham J.A. 1976 ,Astron.J. ,81,687

-Seldner M., Sievers B., Groth E.J. 1977 ,Astron.J. ,82,249

-Shane C.D., Wirtanen C.A. 1967 ,Publ.Lick.Obs. ,XXII,pt.1

-Silk J., Wilson M.L.1985, Proc. of the Int. School of Phys. E. Fermi , Melchiorri F. and Ruffini R. eds.,LXXXVI,431

-Tinsley B.M. 1973 ,Astron.Astrophys. ,266,L79

-Yahil A., Walker D., Rowan-Robinson M. 1986 ,Ap.J.(Letters) ,301,L1 